\title{
Integration of Cloud Computing with Internet of Things: Challenges and Open Issues
}

\author{
Hany F. Atlam ${ }^{1,2}$, Ahmed Alenezi ${ }^{1}$, Abdulrahman Alharthi ${ }^{1}$, Robert J. Walters ${ }^{1}$, and Gary B. Wills ${ }^{1}$ \\ ${ }^{1}$ Electronic and Computer Science Dept., University of Southampton, Southampton, UK \\ ${ }^{2}$ Computer Science and Engineering Dept., Faculty of Electronic Engineering, Menoufia University, Menoufia, Egypt \\ $\{\mathrm{hfa} 1 \mathrm{~g} 15$, aa4e15, aaa2g14, rjw5, gbw\}@soton.ac.uk
}

\begin{abstract}
The Internet of Things (IoT) is becoming the next Internet-related revolution. It allows billions of devices to be connected and communicate with each other to share information that improves the quality of our daily lives. On the other hand, Cloud Computing provides on-demand, convenient and scalable network access which makes it possible to share computing resources; indeed, this, in turn, enables dynamic data integration from various data sources. There are many issues standing in the way of the successful implementation of both Cloud and IoT. The integration of Cloud Computing with the IoT is the most effective way on which to overcome these issues. The vast number of resources available on the Cloud can be extremely beneficial for the IoT, while the Cloud can gain more publicity to improve its limitations with real world objects in a more dynamic and distributed manner. This paper provides an overview of the integration of the Cloud into the IoT by highlighting the integration benefits and implementation challenges. Discussion will also focus on the architecture of the resultant Cloud-based IoT paradigm and its new applications scenarios. Finally, open issues and future research directions are also suggested.
\end{abstract}

Keywords-Cloud Computing, Internet of Things, Cloud based IoT, Integration.

\section{INTRODUCTION}

It is important to explore the common features of the technologies involved in the field of computing. Indeed, this is certainly the case with Cloud Computing and the Internet of Things (IoT) - two paradigms which share many common features. The integration of these numerous concepts may facilitate and improve these technologies. Cloud computing has altered the way in which technologies can be accessed, managed and delivered. It is widely agreed that Cloud computing can be used for utility services in the future [1]. Although many consider Cloud computing to be a new technology, it has, in actual fact, been involved in and encompassed various technologies such as grid, utility computing virtualisation, networking and software services [2], [3]. Cloud computing provides services which make it possible to share computing resources across the Internet. As such, it is not surprising that the origins of Cloud technologies lie in grid, utility computing virtualisation, networking and software services, as well as distributed computing, and parallel computing [4]. On the other hand, the IoT can be considered both a dynamic and global networked infrastructure that manages self-configuring objects in a highly intelligent way. The IoT is moving towards a phase where all items around us will be connected to the Internet and will have the ability to interact with minimum human effort [5]. The IoT normally includes a number of objects with limited storage and computing capacity [6]. It could well be said that Cloud computing and the IoT will be the future of the Internet and next-generation technologies. However, Cloud services are dependent on service providers which are extremely interoperable, while IoT technologies are based on diversity rather than interoperability [6].

This paper provides an overview of the integration of Cloud Computing into the IoT; this involves an examination of the benefits resulting from the integration process and the implementation challenges encountered. Open issues and research directions are also discussed. The remainder of the paper is organised as follows: Section II provides the basic concepts of Cloud computing, IoT, and Cloud-based IoT; Section III discusses the benefits of integrating the IoT into the Cloud; Could-based IoT Architecture is presented in section IV; Section V illustrates different Cloud-based IoT applications scenarios. Following this, the challenges facing Cloud-based IoT integration and open research directions are discussed in Section VI and Section VII respectively, before Section VIII concludes the paper.

\section{BASIC CONCEPTS}

This section reviews the basic concepts of Cloud Computing, the IoT, and Cloud-based IoT.

\section{A. Cloud Computing}

There exist a number of proposed definitions for Cloud computing, although the most widely agreed upon seems be that put forth by the National Institute of Standards and Technology (NIST). Indeed, the NIST has defined Cloud computing as "a model for enabling ubiquitous, convenient, on-demand network access to a shared pool of configurable computing resources (e.g., networks, servers, storage, applications, and services) that can be rapidly provisioned and released with minimal management effort or service provider interaction" [7]. 
As stated in this definition, Cloud computing comprises four types of deployment models, three different service models, and five essential characteristics.

Cloud computing deployment models are most commonly classified as belonging to the public Cloud, where resources are made available to consumers over the Internet. Public Clouds are generally owned by a profitable organisation (e.g. Amazon EC2) [8]. Conversely, the infrastructure of a private Cloud is commonly provided by a single organisation to serve the particular purposes of its users [7]. The private Cloud offers a secure environment and a higher level of control (Microsoft Private Cloud). Hybrid Clouds are a mixture of private and public Clouds. This choice is provided for consumers as it makes it possible to overcome some of the limitations of each model [9]. In contrast, a community Cloud is a Cloud infrastructure which is delivered to a group of users by a number of organisations which share the same need [10].

In order to allow consumers to choose the service that suits them, services in Cloud computing are provided at three different levels, namely: the Software as a Service (SaaS) model, where software is delivered through the Internet to users (e.g. GoogleApps) [2]; the Platform as a Service (PaaS) model, which offers a higher level of integrated environment that can build, test, and deploy specific software (e.g. Microsoft Azure) [11]; and finally, with the Infrastructure as a Service (IaaS) model, infrastructure such as storage, hardware and servers are delivered as a service (e.g. Amazon Web Services) [7].

\section{B. Internet of Things}

The IoT represents a modern approach where boundaries between real and digital domains are progressively eliminated by consistently changing every physical device to a smart alternative ready to provide smart services. All things in the IoT (smart devices, sensors, etc.) have their own identity. They are combined to form the communication network and will become actively participating objects [6]. These objects include not only daily usable electronic devices, but also things like food, clothing, materials, parts, and subassemblies; commodities and luxury items; monuments and landmarks; and various forms of commerce and culture [7]. In addition, these objects are able to create requests and alter their states. Thus, all IoT devices can be monitored, tracked and counted, which significantly decreases waste, loss, and cost [8].

The concept of the IoT was first mentioned by Kevin Ashton in 1999 [9], [10], when he stated that "The Internet of Things has the potential to change the world, just as the Internet did. Maybe even more so". Later, the IoT was formally presented by the International Telecommunication Union (ITU) in 2005 [11]. A great many definitions of the IoT have been put forth by numerous organisations and researchers. According to the ITU (2012), the IoT is "a global infrastructure for the Information Society, enabling advanced services by interconnecting (physical and virtual) things based on, existing and evolving, interoperable information and communication technologies" [12]. The IoT introduces a variety of opportunities and applications. However, it faces many challenges which could potentially hinder its successful implementation, such as data storage, heterogeneous resourceconstrained, scalability, Things, variable geospatial deployment, and energy efficiency [13].

\section{Cloud-Based Internet of Things}

The IoT and Cloud computing are both rapidly developing services, and have their own unique characteristics. On the one hand, the IoT approach is based on smart devices which intercommunicate in a global network and dynamic infrastructure. It enables ubiquitous computing scenarios. The IoT is typically characterised by widely-distributed devices with limited processing capabilities and storage. These devices encounter issues regarding performance, reliability, privacy, and security [14]. On the other hand, Cloud computing comprises a massive network with unlimited storage capabilities and computation power. Furthermore, it provides a flexible, robust environment which allows for dynamic data integration from various data sources [8]. Cloud computing has partially resolved most of the IoT issues. Indeed, the IoT and Cloud are two comparatively challenging technologies, and are being combined in order to change the current and future environment of internetworking services [6].

The Cloud-based Internet of Things is a platform which allows for the smart usage of applications, information, and infrastructure in a cost-effective way. While the IoT and Cloud computing are different from each other, their features are almost complementary, as shown in TABLE 1. This complementarity is the primary reason why many researchers have proposed their integration [14].

TABLE 1. COMPARISON OF THE IOT WITH CLOUD COMPUTING

\begin{tabular}{|l|l|l|}
\hline \multicolumn{1}{|c|}{ Items } & \multicolumn{1}{c|}{ IoT } & \multicolumn{1}{c|}{ Cloud Computing } \\
\hline Characteristics & $\begin{array}{l}\text { IoT is pervasive (things } \\
\text { are everywhere). } \\
\text { These are real world } \\
\text { objects. }\end{array}$ & $\begin{array}{l}\text { Cloud is ubiquitous } \\
\text { (resources are available } \\
\text { from everywhere). } \\
\text { These are virtual resources. }\end{array}$ \\
\hline $\begin{array}{l}\text { Processing } \\
\text { capabilities }\end{array}$ & $\begin{array}{l}\text { Limited computational } \\
\text { capabilities. }\end{array}$ & $\begin{array}{l}\text { Virtually unlimited } \\
\text { computational capabilities. }\end{array}$ \\
\hline $\begin{array}{l}\text { Storage } \\
\text { capabilities }\end{array}$ & $\begin{array}{l}\text { Limited storage or no } \\
\text { storage capabilities. }\end{array}$ & $\begin{array}{l}\text { Unlimited storage } \\
\text { capabilities. }\end{array}$ \\
\hline Connectivity & $\begin{array}{l}\text { It uses the Internet as a } \\
\text { point of convergence. }\end{array}$ & $\begin{array}{l}\text { It uses the Internet for } \\
\text { service delivery. }\end{array}$ \\
\hline Big data & It is a source of big data. & $\begin{array}{l}\text { It is a means by which to } \\
\text { manage big data. }\end{array}$ \\
\hline
\end{tabular}

\section{BENEFITS OF INTEGRATING IOT WITH CLOUD}

Since the IoT suffers from limited capabilities in terms of processing power and storage, it must also contend with issues such as performance, security, privacy, reliability. The integration of the IoT into the Cloud is certainly the best way to overcome most of these issues. The Cloud can even benefit from the IoT by expanding its limits with real world objects in a more dynamic and distributed way, and providing new services for billions of devices in different real life scenarios [6], [14]. In addition, the Cloud provides simplicity of use and reduces the cost of the usage of applications and services for end-users. The Cloud also simplifies the flow of the IoT data gathering and processing, and provides quick, low-cost installation and integration for complex data processing and 
deployment [15]. The benefits of integrating IoT into Cloud are discussed in this section as follows.

\section{Communication}

Application and data sharing are two significant features of the Cloud-based IoT paradigm. Ubiquitous applications can be transmitted through the IoT, whilst automation can be utilised to facilitate low-cost data distribution and collection. The Cloud is an effective and economical solution which can be used to connect, manage, and track anything by using built-in apps and customised portals [7]. The availability of fast systems facilitates dynamic monitoring and remote objects control, as well as data real-time access. It is worth declaring that, although the Cloud can greatly develop and facilitate the IoT interconnection, it still has weaknesses in certain areas. Thus, practical restrictions can appear when an enormous amount of data needs to be transferred from the Internet to the Cloud [7], [16].

\section{Storage}

As the IoT can be used on billions of devices, it comprises a huge number of information sources, which generate an enormous amount of semi-structured or non-structured data [17]. This is known as Big Data, and has three characteristics [4]: variety (e.g. data types), velocity (e.g. data generation frequency), and volume (e.g. data size). The Cloud is considered to be one of the most cost-effective and suitable solutions when it comes to dealing with the enormous amount of data created by the IoT. Moreover, it produces new chances for data integration, aggregation, and sharing with third parties [18].

\section{Processing capabilities}

IoT devices are characterised by limited processing capabilities which prevent on-site and complex data processing. Instead, gathered data is transferred to nodes that have high capabilities; indeed, it is here that aggregation and processing are accomplished. However, achieving scalability remains a challenge without an appropriate underlying infrastructure. Offering a solution, the Cloud provides unlimited virtual processing capabilities and an on-demand usage model [18]. Predictive algorithms and data-driven decisions making can be integrated into the IoT in order to increase revenue and reduce risks at a lower cost [6].

\section{Scope}

With billions of users communicating with one another together and a variety of information being collected, the world is quickly moving towards the Internet of Everything (IoE) realm - a network of networks with billions of things that generate new chances and risks [18]. The Cloud-based IoT approach provides new applications and services based on the expansion of the Cloud through the IoT objects, which in turn allows the Cloud to work with a number of new real world scenarios, and leads to the emergence of new services [22].

\section{New abilities}

The IoT is characterised by the heterogeneity of its devices, protocols, and technologies. Hence, reliability, scalability, interoperability, security, availability and efficiency can be very hard to achieve. Integrating IoT into the Cloud resolves most of these issues [6]. It provides other features such as easeof-use and ease-of-access, with low deployment costs [19], [22].

\section{New Models}

Cloud-based IoT integration empowers new scenarios for smart objects, applications, and services [11], [20]. Some of the new models are listed as follows:

- SaaS (Sensing as a Service) [11], which allows access to sensor data;

- EaaS (Ethernet as a Service) [23], the main role of which is to provide ubiquitous connectivity to control remote devices;

- SAaaS (Sensing and Actuation as a Service) [11], which provides control logics automatically.

- IPMaaS (Identity and Policy Management as a Service) [23], which provides access to policy and identity management.

- DBaaS (Database as a Service) [23], which provides ubiquitous database management;

- SEaaS (Sensor Event as a Service) [11], which dispatches messaging services that are generated by sensor events;

- SenaaS (Sensor as a Service) [23], which provides management for remote sensors;

- DaaS (Data as a Service) [23], which provides ubiquitous access to any type of data.

\section{Cloud-BAsed IoT ARCHITECTURE}

According to a number of previous studies, the well-known IoT architecture is typically divided into three different layers: application, perception and network layer. Most assume that the network layer is the Cloud layer, which realises the Cloudbased IoT architecture, as depicted in Fig. 1.

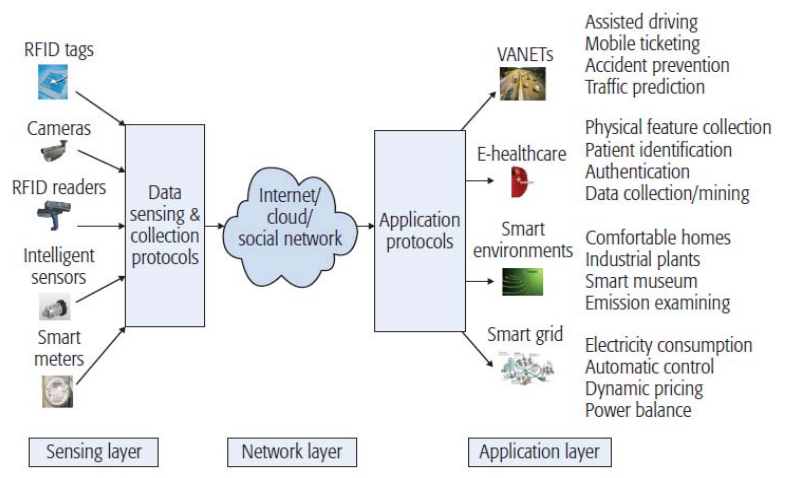

Fig. 1. Cloud-based IoT architecture [1]. 
The perception layer is used to identify objects and gather data, which is collected from the surrounding environment. In contrast, the main objective of the network layer is to transfer the collected data to the Internet/Cloud. Finally, the application layer provides the interface to different services [20].

\section{ClOUd-BASED IOT APPLICATIONS}

The Cloud-based IoT approach has introduced a number of applications and smart services, which have affected end users' daily lives. TABLE 2 presents a brief discussion of certain applications which have been improved by the Cloud-based IoT paradigm [4], [24 - 27].

\section{TABLE 2. CLOUD-BASED IOT APPLICATIONS}

\begin{tabular}{|c|c|}
\hline $\begin{array}{c}\text { Application } \\
\text { Field } \\
\end{array}$ & Description \\
\hline Healthcare & $\begin{array}{l}\text { Cloud-based IoT has brought many benefits and } \\
\text { opportunities to the field of healthcare. It can } \\
\text { clearly develop and improve healthcare services } \\
\text { and keep the field innovative (e.g. intelligent } \\
\text { drug/medicine control, hospital management). }\end{array}$ \\
\hline Smart Cities & $\begin{array}{l}\text { A middleware for future smart cities can be } \\
\text { provided through the IoT, attaining data from } \\
\text { sensing infrastructure, IoT technologies and placing } \\
\text { information in a consistent manner. This will lead } \\
\text { to the generation of services that can communicate } \\
\text { with the surrounding environments (e.g. Smart } \\
\text { streetlights, Bigbelly, ShotSpotte). }\end{array}$ \\
\hline Smart Homes & $\begin{array}{l}\text { A large number of Cloud-based IoT applications } \\
\text { have enabled the automation of home activities, } \\
\text { where the adoption of various embedded devices } \\
\text { and Cloud computing has empowered the } \\
\text { automation of in-house activities (e.g. home } \\
\text { security control, smart metering, energy saving). }\end{array}$ \\
\hline $\begin{array}{l}\text { Video } \\
\text { surveillance }\end{array}$ & $\begin{array}{l}\text { By embracing Cloud-based IoT, intelligent video } \\
\text { surveillance will make it possible to manage, store } \\
\text { and process video content from video sensors } \\
\text { easily and efficiently; this will also make it possible } \\
\text { to extract information from scenes automatically. It } \\
\text { has become one of the supreme tools for many } \\
\text { security-related applications (e.g. Wireless CCTV } \\
\text { Cameras, Movement detection system). }\end{array}$ \\
\hline $\begin{array}{l}\text { Automotive and } \\
\text { Smart Mobility }\end{array}$ & $\begin{array}{l}\text { The integration of Cloud computing into The } \\
\text { Global Positioning System (GPS) and other } \\
\text { transportation technologies represents a promising } \\
\text { opportunity to solve many of the existing } \\
\text { challenges (e.g. traffic state prediction \& } \\
\text { notification, remote vehicles). }\end{array}$ \\
\hline $\begin{array}{l}\text { Smart energy and } \\
\text { smart grid }\end{array}$ & $\begin{array}{l}\text { Cloud computing and the IoT can work together } \\
\text { effectively to provide consumers with smart } \\
\text { management of energy consumption (e.g. smart } \\
\text { meters, smart appliances, renewable energy } \\
\text { resources). }\end{array}$ \\
\hline Smart logistics & $\begin{array}{l}\text { It allows for, and eases, the automated management } \\
\text { of goods flow between producers and consumers, } \\
\text { while simultaneously enabling the tracking of } \\
\text { goods in transit (e.g. logistics industry, tracking } \\
\text { shipments). }\end{array}$ \\
\hline $\begin{array}{l}\text { Environmental } \\
\text { monitoring }\end{array}$ & $\begin{array}{l}\text { By combining the Cloud with the IoT, a high-speed } \\
\text { information system can be provided which will link } \\
\text { the entity that monitors wide-area environments } \\
\text { and sensors that have been properly deployed in the } \\
\text { area (e.g. pollution source monitoring, water } \\
\text { quality monitoring, air quality monitoring). }\end{array}$ \\
\hline
\end{tabular}

\section{CHALLENGES FACING CLOUD-BASED IOT INTEGRATION}

There are many challenges which could potentially prevent the successful integration of the Cloud-based IoT paradigm. These challenges include:

\section{Security and privacy.}

Cloud-based IoT makes it possible to transport data from the real world to the Cloud. Indeed, one particularly important issues which has not yet been resolved is how to provide appropriate authorisation rules and policies while ensuring that only authorised users have access to the sensitive data; this is crucial when it comes to preserving users' privacy, and particularly when data integrity must be guaranteed [18]. In addition, when critical IoT applications move into the Cloud, issues arise because of the lack of trust in the service provider, information regarding service level agreements (SLAs), and the physical location of data [25], [27]. Sensitive information leakage can also occur due to the multi-tenancy. Moreover, public key cryptography cannot be applied to all layers because of the processing power constraints imposed by IoT objects [18]. New challenges also require specific attention; for example, the distributed system is exposed to number of possible attacks, such as SQL injection, session riding, crosssite scripting, and side-channel. Moreover, important vulnerabilities, including session hijacking and virtual machine escape are also problematic [18], [29].

\section{Heterogeneity}

One particularly important challenge faced by the Cloudbased IoT approach is related to the extensive heterogeneity of devices, platforms, operating systems, and services that exist and might be used for new or developed applications. Cloud platforms suffer from heterogeneity issues; for instance, Cloud services generally come with proprietary interfaces, thus allowing for resource integration based on specific providers [18]. In addition, the heterogeneity challenge can be exacerbated when end-users adopt multi-Cloud approaches, and thus services will depend on multiple providers to improve application performance and resilience [30].

\section{Big data}

With many predicting that Big Data will reach 50 billion IoT devices by 2020 , it is important to pay more attention to the transportation, access, storage and processing of the enormous amount of data which will be produced. Indeed, given recent technological developments, it is clear that the IoT will be one of the core sources of big data, and that the Cloud can facilitate the storage of this data for a long period of time, in addition to subjecting it to complex analysis [4]. Handling the huge amount of data produced is a significant issue, as the application's whole performance is heavily reliant on the properties of this data management service. Finding a perfect data management solution which will allow the Cloud to manage massive amounts of data is still a big issue [31]. Furthermore, data integrity is a vital element, not only because of its effect on the service's quality, but also because of security and privacy issues, the majority of which relate to outsourced data [18]. 


\section{Performance}

Transferring the huge amount of data created from IoT devices to the Cloud requires high bandwidth. As a result, the key issue is obtaining adequate network performance in order to transfer data to Cloud environments; indeed, this is because broadband growth is not keeping pace with storage and computation evolution [18]. In a number of scenarios, services and data provision should be achieved with high reactivity [29]. This is because timeliness might be affected by unpredictable matters and real-time applications are very sensitive to performance efficiency [18].

\section{Legal aspects}

Legal aspects have been very significant in recent research concerning certain applications. For instance, service providers must adapt to various international regulations. On the other hand, users should give donations in order to contribute to data collection [32].

\section{Monitoring}

Monitoring is a primary action in Cloud Computing when it comes to performance, managing resources, capacity planning, security, SLAs, and for troubleshooting. As a result, the Cloudbased IoT approach inherits the same monitoring demands from the Cloud, although there are still some related challenges that are impacted by velocity, volume, and variety characteristics of the IoT [4], [31].

\section{Large scale}

The Cloud-based IoT paradigm makes it possible to design new applications that aim to integrate and analyse data coming from the real world into IoT objects. This requires interacting with billions of devices which are distributed throughout many areas [28]. The large scale of the resulting systems raises many new issues that are difficult to overcome. For instance, achieving computational capability and storage capacity requirements is becoming difficult. Moreover, the monitoring process has made the distribution of the IoT devices more difficult, as IoT devices have to face connectivity issues and latency dynamics [18].

\section{OPEN ISSUES AND RESEARCH DIRECTIONS}

This section will address some of the open issues and future research directions related to Cloud-based IoT, and which still require more research efforts. These issues include:

\section{Standardisation}

Many studies have highlighted the issues of lack of standards, which is considered critical in relation to the Cloudbased IoT paradigm [18]. Although a number of proposed standardisations have been put forth by the scientific society for the deployment of IoT and Cloud approaches, it is obvious that architectures, standard protocols, and APIs are required to allow for interconnection between heterogeneous smart things and the generation of new services, which make up the Cloudbased IoT paradigm [4], [6], [18].

\section{Fog Computing}

Fog computing is a model which extends Cloud computing services to the edge of the network. Similar to the Cloud, Fog supply communicates application services to users. Fog can essentially be considered an extension of Cloud Computing which acts as an intermediate between the edge of the network and the Cloud; indeed, it works with latency-sensitive applications that require other nodes to satisfy their delay requirements [6]. Although storage, computing, and networking are the main resources of both Fog and the Cloud, the Fog has certain features, such as location awareness and edge location, that provide geographical distribution, and low latency; moreover, there are a large nodes; this is in contrast with the Cloud, which is supported for real-time interaction and mobility [4], [6].

\section{Cloud Capabilities}

As in any networked environment, security is considered to be one of the main issues of the Cloud-based IoT paradigm. There are more chances of attacks on both the IoT and the Cloud side. In the IoT context, data integrity, confidentiality and authenticity can be guaranteed by encryption. However, insider attacks cannot be resolved and it is also hard to use the IoT on devices with limited capabilities [4], [18].

\section{SLA enforcement}

Cloud-based IoT users need created data to be conveyed and processed based on application-dependent limitations, which can be tough in some cases. Ensuring a specific Quality of Service (QoS) level regarding Cloud resources by depending on a single provider raises many issues. Thus, multiple Cloud providers may be required to avoid SLA violations. However, dynamically choosing the most appropriate mixture of Cloud providers still represents an open issue due to time, costs, and heterogeneity of QoS management support [18], [31].

\section{Big data}

In the previous section, we discussed Big Data as a critical challenge that is tightly coupled with the Cloud-based IoT paradigm. Although a number of contributions have been proposed, Big Data is still considered a critical open issue, and one in need of more research. The Cloud-based IoT approach involves the management and processing of huge amounts of data stemming from various locations and from heterogeneous sources; indeed, in the Cloud-based IoT, many applications need complicated tasks to be performed in real-time [18], [28].

\section{Energy efficiency}

Recent Cloud-based IoT applications include frequent data that is transmitted from IoT objects to the Cloud, which quickly consumes the node energy. Thus, generating efficient energy when it comes to data processing and transmission remains a significant open issue [4]. Several directions have been suggested to overcome this issue, such as compression technologies, efficient data transmission; and data caching techniques for reusing collected data with time-insensitive application [18], [31]. 


\section{Security and privacy}

Although security and privacy are both critical research issues which have received a great deal of attention, they are still open issues which require more efforts. Indeed, adapting to different threats from hackers is still an issue [4], [30]. Moreover, another problem is providing the appropriate authorisation rules and policies while ensuring that only authorised users have access to sensitive data; this is crucial for preserving users' privacy, specifically when data integrity must be guaranteed [18].

\section{CONCLUSION}

The IoT is becoming an increasingly ubiquitous computing service which requires huge volumes of data storage and processing capabilities. The IoT has limited capabilities in terms of processing power and storage, while there also exist consequential issues such as security, privacy, performance, and reliability; As such, the integration of the Cloud into the IoT is very beneficial in terms of overcoming these challenges. In this paper, we presented the need for the creation of the Cloud-based IoT approach. Discussion also focused on the Cloud-based IoT architecture, different applications scenarios, challenges facing the successful integration, and open research directions. In future work, a number of case studies will be carried out to test the effectiveness of the Cloud-based IoT approach in healthcare applications.

\section{REFERENCES}

[1] R. Buyya, C. Shin, S. Venugopal, J. Broberg, and I. Brandic, "Cloud computing and emerging IT platforms: Vision, hype, and reality for delivering computing as the 5th utility," Futur. Gener. Comput. Syst., 2009, pp. 599-616.

[2] M. Armbrust, A. Fox, R. Griffith, A. D. Joseph, R. Katz, A. Konwinski, G. Lee, D. Patterson, A. Rabkin, and I. Stoica, "A View of Cloud Computing," Commun. ACM, vol. 53, no. 4, 2010, pp. 50-58.

[3] K. Gai, "Towards Cloud Computing: A Literature Review on Cloud Computing and its Development Trends," 2012 Fourth Int. Conf. Multimed. Inf. Netw. Secur., 2012, pp. 142-146.

[4] A. Botta, W. de Donato, V. Persico and A. Pescapé, "On the Integration of Cloud Computing and Internet of Things," 2014 International Conference on Future Internet of Things and Cloud, Barcelona, 2014, pp. 23-30.

[5] R. Shanbhag and R. Shankarmani, "Architecture for Internet of Things to minimize human intervention," 2015 Int. Conf. Adv. Comput. Commun. Informatics, 2015, pp. 2348-2353.

[6] S. M. Babu, A. J. Lakshmi and B. T. Rao, "A study on cloud based Internet of Things: CloudIoT," 2015 Global Conference on Communication Technologies (GCCT), 2015, pp. 60-65.

[7] B. B. P. Rao, P. Saluia, N. Sharma, A. Mittal and S. V. Sharma, "Cloud computing for Internet of Things \& sensing based applications," 2012 Sixth International Conference on Sensing Technology (ICST), Kolkata, 2012, pp. 374-380.

[8] J. Zhou et al., "CloudThings: A common architecture for integrating the Internet of Things with Cloud Computing," Proceedings of the 2013 IEEE 17th International Conference on Computer Supported Cooperative Work in Design (CSCWD), 2013, pp. 651-657.

[9] K. Ashton, “That 'Internet of Things' Thing," RFiD J., 2009, pp. 49-86.

[10] G. Joshi and S. Kim, "Survey, Nomenclature and Comparison of Reader Anti-Collision Protocols in RFID," IETE Tech. Rev., vol. 25, no. 5, 2013, pp. 285-292.

[11] ITU, "The Internet of Things," Itu Internet Rep., 2005, pp. 114-137.

[12] ITU, "Overview of the Internet of things", 2012, pp. 22-40.
[13] F. Kawsar, G. Kortuem, and B. Altakrouri, "Supporting Interaction with the Internet of Things across Objects , Time and Space," 2010.

[14] A. Botta, W. De Donato, V. Persico, and A. Pescapé, "Integration of Cloud computing and Internet of Things: A survey," Futur. Gener. Comput. Syst., vol. 56, 2016, pp. 684-700.

[15] Dash, Sanjit Kumar, Subasish Mohapatra, and Prasant Kumar Pattnaik. "A survey on applications of wireless sensor network using cloud computing." International Journal of Computer science \& Engineering Technologies, 2010, pp. 50-55.

[16] G. C. Fox, S. Kamburugamuve and R. D. Hartman, "Architecture and measured characteristics of a cloud based internet of things," 2012 International Conference on Collaboration Technologies and Systems (CTS), 2012, pp. 6-12.

[17] S. Aguzzi D. Bradshaw M. Canning M. Cansfield P. Carter G. Cattaneo S. Gusmeroli G. Micheletti D. Rotondi R. Stevens "Definition of a Research and Innovation Policy Leveraging Cloud Computing and IoT Combination. Final Report" European Commission SMART, 2013.

[18] G. Suciu A. Vulpe S. Halunga O. Fratu G. Todoran V. Suciu "Smart Cities Built on Resilient Cloud Computing and Secure Internet of Things" 19th Int. Conf. Control Systems and Computer Science (CSCS) 2013 , pp. 513-518.

[19] [1] A. Zaslavsky, C. Perera, and D. Georgakopoulos, "Sensing as a Service and Big Data," arXiv Prepr., 2013.

[20] M. Wu, T. Lu, F. Ling, and H. Du, "Research on the architecture of Internet of things," 2010 3rd Int. Conf. Adv. Comput. Theory Eng., vol. 5, 2010, pp. 484-487.

[21] I. Lee and K. Lee, "The Internet of Things ( IoT ): Applications , investments, and challenges for enterprises," Bus. Horiz., vol. 58, no. 4, 2015, pp. 431-440.

[22] S. Chen, S. Member, H. Xu, D. Liu, S. Member, B. Hu, and H. Wang, "A Vision of IoT: Applications, Challenges, and Opportunities With China Perspective," IEEE INTERNET THINGS J., vol. 1, no. 4, 2014, pp. 349-359.

[23] P. Parweker, "From Internet of things toward Cloud of Things," Int. Conf. Comput. Commun. Technol., 2011, pp. 329-333.

[24] S. M. Babu, A. J. Lakshmi, and B. T. Rao, "A study on cloud based Internet of Things: CloudIoT," 2015 Glob. Conf. Commun. Technol., no. Gcct, 2015, pp. 60-65.

[25] A. Alenezi, N. H. N. Zulkipli, H. F. Atlam, R. J. Walters, and G. B. Wills, "The Impact of Cloud Forensic Readiness on Security," in 7st International Conference on Cloud Computing and Services Science, 2017, pp. 1-8.

[26] J. Zhou, Z. Cao, X. Dong, and A. V Vasilakos, "Security and Privacy for Cloud-Based IoT: Challenges , Countermeasures, and Future Directions," no. January, 2017, pp. 26-33.

[27] K. S. Dar, A. Taherkordi and F. Eliassen, "Enhancing Dependability of Cloud-Based IoT Services through Virtualization," 2016 IEEE First International Conference on Internet-of-Things Design and Implementation (IoTDI), 2016, pp. 106-116.

[28] Díaz, Manuel, Cristian Martín, and Bartolomé Rubio. "State-of-the-art, challenges, and open issues in the integration of Internet of things and cloud computing." Journal of Network and Computer Applications, 2016, pp. 99-117.

[29] C. Doukas and I. Maglogiannis, "Bringing IoT and cloud computing towards pervasive healthcare," Proc. - 6th Int. Conf. Innov. Mob. Internet Serv. Ubiquitous Comput. IMIS, 2012, pp. 922-926.

[30] H. F. Atlam, A. Alenezi, R. J. Walters, and G. B. Wills, "An Overview of Risk Estimation Techniques in Risk-based Access Control for the Internet of Things," in 2nd International Conference on Internet of Things, Big Data and Security, 2017, pp. 1-8. 